\title{
CRECIMIENTO DE PLANTACIONES CON RAULI Y ROBLE BAJO DOSEL EN DEPENDENCIA DEL GRADO DE LUMINOSIDAD Y FERTILIZACION
}

Hans Grosse W.(*)

\section{RESUMEN}

En un rodal del tipo forestal Roble-Rauli-Coigüe, en el fundo Jauja en los $38^{\circ} 13^{\prime} 51^{\prime \prime}$ Lat. Sur, $72^{\circ} 0^{\prime} 38^{\prime \prime}$ Long. Oeste a 790 m.s.n m. se instaló un ensayo de plantación para las especies raulí y roble bajo dosel y en terreno descubierto.

Se consideraron los factores área basal en cinco niveles $(0$. $13-28-38-69 \mathrm{~m}^{2} / \mathrm{ha}$ ) y fertilizante (NPK) con y sin aplicación.

La sobrevivencia fue de un $80 \%$ a $90 \%$ en la situación con $13 \mathrm{~m}^{2}$ /ha de $\mathrm{AB}$, del $0 \%$ bajo la situación extrema con $69 \mathrm{~m}^{2} / \mathrm{ha}$ de $\mathrm{AB}$ y del $40 \%$ a $65 \%$ en la situación sin árboles para raulí y roble, respectivamente.

A medida que disminuía el área basal y se producía así una mayor disponibilidad de luz, aumentaron los incrementos para las variables de estado diámetro, altura y volumen. El roble reaccionó con mayor sensibilidad a una mayor disponibilidad de luz, que el raulí.

Ambas especies aumentaron sus niveles de sobrevivencia y crecimiento al aplicar fertilizante.

Los incrementos más altos se lograron para las plantas fertilizadas en el área sin árboles. Estos fueron $24 \mathrm{~cm}$ y $75 \mathrm{~cm}$ de crecimiento en altura para raulí y roble respectivamente.

\section{ABSTRACT}

A complete factorial test installed as a split-plot was used to determine the effect of 1) basal area (levels: $0 \% \mathrm{~m}^{2}, 13 \mathrm{~m}^{2}$, $28 \mathrm{~m}^{2}, 38 \mathrm{~m}^{2}$ and $69 \mathrm{~m}^{2}$ ) in combination with 2) fertilization (levels: with NPK and without NPK) on the species rauli (Nothofagus alpina) and roble (Nothofagus obliqua). The site was a roblerauli-coigüe mixed forest, 790 meters above sea level, latitude $38^{\circ}$ $13^{\prime} 51^{\prime \prime} \mathrm{S}$ and longitude $72^{\circ} 0^{\prime} 38^{\prime \prime} \mathrm{W}$.

There was an $80 \%-90 \%$ survival under the $13 \mathrm{~m}^{2}$ basal area $0 \%$ survival under the $69 \mathrm{~m}^{2}$ basal area situation und $40 \%$ to $65 \%$ survival in the situation without trees for rauli and roble respectively.

Less basal area resulted in increasing the diameter, height and a volume index and also seemed to increase more the growth of roble than rauli.

Both species had higher survival rates, height and diameter growth with fertilizers. The best height growth increase was 24 $\mathrm{cm}$ for rauli and $75 \mathrm{~cm}$ for roble under full light conditions in the situation without trees.

(*) Ingeniero Forestal Dr. División Regional, Instituto Forestal, Barros Arana 121. Concepción - Chile. 


\section{INTRODUCCION}

Los bosques nativos chilenos fueron hasta fines de los afoos 60 el pilar de la producción maderera nacional. Repetidos floreos, quemas y cortas a tala rasa los empobrecieron en cuanto a la participación de sus especies más valiosas. A esto se debe que la regeneración natural, posterior a explotaciones, en gran medida se instale con individuos de menor valor comercial.

El deterioro en el valor del recurso por la integración mayoritaria de especies de menor valor o bien individuos de mala calidad, hace urgente recuperar su productividad. Esto se puede lograr a través del "enriquecimiento del bosque" con las especies de mayor valor comercial a través de la repoblación artificial. Este método permite seleccionar los árboles productores de semillas para así garantizar desde el vivero plantas competitivas y de un valor potencial interesante. Estas se integrarán a rodales semiabiertos y bajo la competencia de árboles remanentes vivos y anillados. Por este motivo es indispensable conocer el comportamiento que se puede obtener con diversas especies bajo la situación de competencia. La proyección productiva que pueda tener este tipo de repoblación queda en evidencia con las plantaciones de rauli y otras especies nativas realizadas en los años setenta y ochenta entre los lagos Pirihueico, Panguipulli y Riñihue. (GROSSE, 1987; GROSSE y BOURKE, 1987).

En el presente artículo se presentan resultados de plantaciones experimentales realizadas con las especies raulí y roble, en rodales con cinco niveles de área basal, con y sin fertilización. Los resultados corresponden al desarrollo que han alcanzado las plantas después de su primer período vegetativo en terreno.

El objetivo de estas plantaciones experimentales es obtener y entregar antecedentes concretos acerca del desarrollo que se puede esperar de enriquecimiento con raulí y roble, bajo distintas situaciones de competencia y luminosidad.

\section{ANTECEDENTES GENERALES DEL SECTOR EN ESTUDIO}

El ensayo se realizó en el fundo Jauja, sector Santa Luisa en los $38^{\circ} 13^{\prime} 51^{\prime \prime}$ Lat. Sur, $72^{\circ}$ 0' $38^{\prime \prime}$ Long. Oeste a $790 \mathrm{~m} . \mathrm{s} \mathrm{n} \mathrm{m}$. Se encuentra a $63 \mathrm{~km}$ al este del pueblo de Collipulli en la provincia de Malleco de la IX Región.

\section{Caracteristicas de los suelos}

Los suelos del área están formados por cenizas volcánicas que descansan usualmente sobre conglomerados o tobas volcánicas, andesitas o basaltos. Pertenecen a la serie Santa Bárbara, cuyas características son: Textura moderadamente liviana, suelo profundo, alto tenor de materia orgánica, estructura granular, buen drenaje, alta capacidad de retención de agua, PH 5 a 6 ; fertilidad media a baja con un alto poder de fijación de fósforo (PERALTA, 1976).

\section{Antecedentes climáticos}

Los antecedentes climáticos recopilados en el lugar más cercano al sector del ensayo corresponden a la estación meteorológica "Jauja". Esta se encuentra aproximadamente a $14 \mathrm{~km}$ del sector del ensayo a 450 m.s.n.m. Como la plantación experimental se ubicó en un lugar más alto, a 790 m.s.n.m., la información recopilada en la estación meteorológica Jauja no se puede extrapolar directamente al lugar del ensayo. Su utilidad para éste consiste en que proporciona información sobre las tendencias climáticas para el área precordillerana considerada. Es de suponer que los niveles de temperatura en el lugar de la plantación son menores que en Jauja. 
Entre el año 1978 y 1987 la precipitación media anual registrada fue de $2.522 \mathrm{~mm}$. En la Tabla 1 se entregan antecedentes históricos mensuales para los meses que duró el período de observación, agregando los registros de temperaturas.

En términos generales los registros de agua caída durante el período de observación siguen la tendencia de los niveles históricos. Las excepciones se dieron en el mes de Febrero, donde no hubo precipitación y en Marzo, duplicándose el promedio histórico.

TABLA 1

ANTECEDENTES METEOROLOGICOS REGISTRADOS EN LA ESTACION JAUJA
DURANTE EL PERIODO DE OBSERVACION

(Agosto de 1987 a Abril de 1988)

\begin{tabular}{|c|c|c|c|c|c|c|c|c|}
\hline \multirow[b]{2}{*}{ MESES } & \multicolumn{6}{|c|}{ TEMPERATURA $\left({ }^{\circ} \mathrm{C}\right)$} & \multicolumn{2}{|c|}{$\begin{array}{c}\text { PLUVIOMETRIA (mm) } \\
\text { TOTAL }\end{array}$} \\
\hline & $\begin{array}{l}\text { MIN. } \\
\text { MED. }\end{array}$ & $\begin{array}{l}\text { MAX. } \\
\text { MED. }\end{array}$ & $\underset{\text { MES }}{\text { MEDIA }}$ & $\begin{array}{l}\text { MIN. } \\
\text { ABS. }\end{array}$ & $\begin{array}{l}\text { MAX. } \\
\text { ABS. }\end{array}$ & $\begin{array}{l}\text { DIAS } \\
\text { HEL. }\end{array}$ & $\begin{array}{c}\text { MES } \\
(1987-88)\end{array}$ & $\begin{array}{c}\text { MES } \\
(1978-87)\end{array}$ \\
\hline AGO. & 2,8 & 14,2 & 8,5 & $-1,0$ & 25,0 & 8 & 344,0 & 282,5 \\
\hline SEP. & 3,1 & 16,6 & 9,9 & $-1,0$ & 26,0 & 4 & 183,0 & 222,8 \\
\hline OCT. & 5,5 & 19,5 & 12,5 & 1,0 & 27,0 & 0 & 135,0 & 173,5 \\
\hline NOV. & 6,7 & 22,5 & 14,6 & 2,0 & 29,0 & 0 & 86,0 & 148,0 \\
\hline DIC. & 6,8 & 23,8 & 15,3 & 3,0 & 30,0 & 0 & 50,0 & 41,7 \\
\hline ENER. & 6,8 & 25,3 & 16,1 & 2,0 & 22,0 & 0 & 96,0 & 74,6 \\
\hline FEBR. & 5,4 & 29,1 & 17,3 & 3,0 & 37,0 & 0 & 0,0 & 63,1 \\
\hline MAR. & 7,4 & 22,7 & 15,1 & 2,0 & 30,0 & 0 & 119,0 & 63,0 \\
\hline ABR. & 5,2 & 20,2 & 12,7 & 2,0 & 32,0 & 0 & 142,0 & 161,1 \\
\hline
\end{tabular}

Fuente: FORVESA S.A.

\section{Tipo forestal}

Los rodales del área en estudio corresponden al tipo forestal roble-raulí-coigüe según la clasificación de DONOSO (1981).

El bosque está compuesto por Nothofagus obliqua "roble" y Nothofagus alpina, "raulí" en el dosel superior. Como especies acompañantes se presentan principalmente Persea lingue, "lingue", Aextoxicon punctatum, "olivillo", Lomatia hirsuta "radal" y Gevuina avellana, "avellano".

\section{ANTECEDENTES METODOLOGICOS}

A continuación se entregan antecedentes referidos al diseño experimental, a la situación del rodal, donde se realiza el ensayo, antes y después de la intervención y el material utilizado en la plantación. 


\section{El diseño del ensayo}

Para realizar el ensayo se eligió un área de aproximadamente 2 ha de características homogéneas en cuanto a su composición florística y sitio. Se planteó un experimento factorial, con un diseño en bloques aleatorios con parcelas subdivididas (figura 1). El número de parcelas es de 60. ( $5 \times 2 \times 2 \times 3$ bloques). Cada una consta de 100 plantas de raulí o de roble, considerando una distancia de plantación de $1 \times 1 \mathrm{~m}$.

Los factores y niveles considerados fueron:

Factor área basal $\left(\mathrm{m}^{2} / \mathrm{ha}\right)$ cobertura de copas $(\%)$

$$
\begin{aligned}
& \mathrm{A}_{1}: \quad \mathrm{AB}=0,0 ; \text { Cob. }=00,0 \\
& \mathrm{~A}_{2}: \mathrm{AB}=13,3 ; \text { Cob. }=34,8 \\
& \mathrm{~A}_{3}: \mathrm{AB}=28,2 ; \text { Cob. }=63,1 \\
& \mathrm{~A}_{4}: \mathrm{AB}=37,5 ; \text { Cob. }=87,5 \\
& \mathrm{~A}_{5}: \mathrm{AB}=66,5 ; \text { Cob. }=98,0
\end{aligned}
$$

* Cobertura de copas: Se definió como el área cubierta por la sumatoria de la proyección de las copas para cada una de las situaciones de área başal $\left(A_{1}\right.$ a $\left.A_{s}\right)$. (Figura 1$)$.

Factor especie (B)

$$
\begin{aligned}
& B_{1} \text { : raulí } \\
& B_{2} \text { : roble }
\end{aligned}
$$

Factor fertilizante (C)

$\mathrm{C}_{1}: \sin$

$\mathrm{C}_{2}$ : NPK (Nitrato de amonio $-13 \mathrm{~g} /$ planta, Super-fosfato triple - $10 \mathrm{~g} /$ planta, Salitre potásico $-10 \mathrm{~g} /$ planta). Fecha de aplicación: 14 y 15 de Octubre de 1987.

Las variables de estado evaluadas fueron:

\section{- Sobrevivencia}

- Incremento en altura total (para todas las plantas y para el $10 \%$ con el mejor desarrollo.

- Diámetro del cuello (a $2 \mathrm{~cm}$ desde el nivel del suelo)

- Indicador del volumen $\left(\widetilde{\mathrm{II}} / 3 \cdot \mathrm{r}^{2} \cdot \mathrm{H}\right)$, donde $\mathrm{r}=$ radio a $2 \mathrm{~cm}$ desde el nivel del suelo y $\mathrm{H}=$ altura de la planta). 


\section{FIGURA 1}

\section{DISTRIBUCION DE LAS PARCELAS EN EL ENSAYO DE PLANTACION CON RAULI Y ROBLE}

\section{(Fundo Jauja, sector Santa Luisa)}
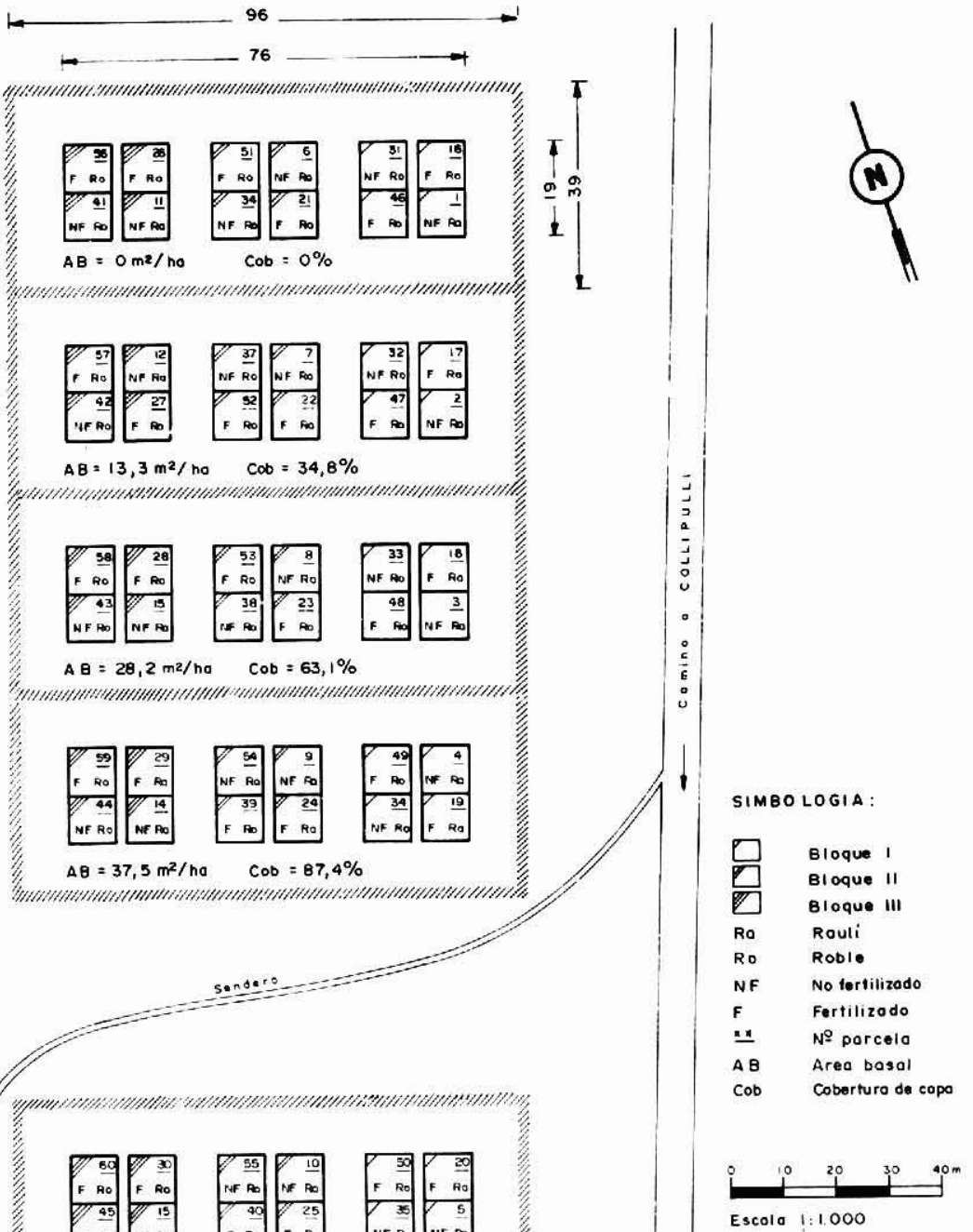

$A B=13,3 \mathrm{~m}^{2} /$ ho $\quad \operatorname{cob}=34,8 \%$

"Y
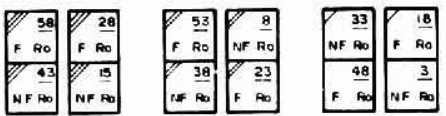

$A B=28,2 \mathrm{~m}^{2} / \mathrm{ho}$

$\operatorname{cob}=63,1 \%$

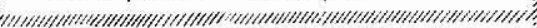
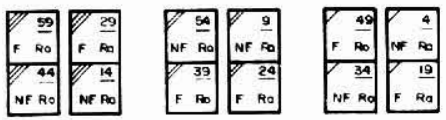

$\Delta B=37,5 \mathrm{~m}^{2} / \mathrm{ho}$

Cob $=87,4 \%$

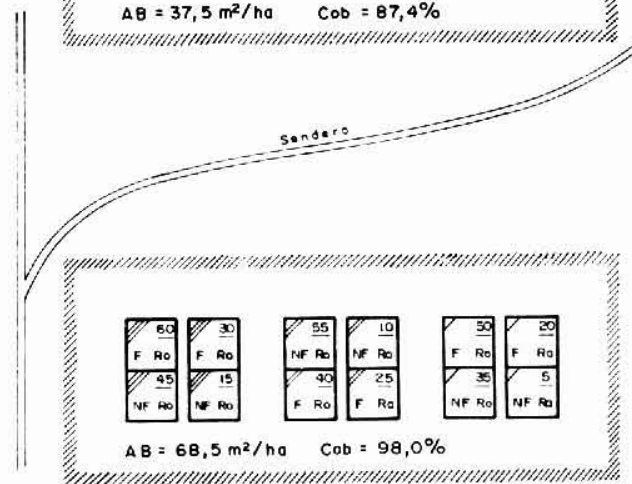




\section{La situación antes y después de la intervención}

Antes que se realizara la intervención, se evaluaron las variables de estado para la situación en general y para los sectores específicos en los cuales se incertaron las parcelas (Tabla 1).

La ocupación del sitio, expresada en área basal, fluctuaba entre los $54 \mathrm{~m}^{2} /$ ha y los $69 \mathrm{~m}^{2} / \mathrm{ha}$ y el número de árboles entre aproximadamente 3.900 y 5.700/ha. El promedio general fue de 5.204 árboles $/ \mathrm{ha}, 62,4 \mathrm{~m}^{2}$ de área basal por ha y $11,4 \mathrm{~cm}$ de diámetro medio aritmético.

Más de un $50 \%$ del área basal correspondia a raulí y roble. En orden de importancia le segu ían lingue, laurel, avellano, mañio y arrayán.

En término de número de árboles, raulí y roble sólo presentaban el $25 \%$. El resto lo constituían las especies de mayor tolerancia a la sombra.

TABLA 1

NUMERO DE ARBOLES, AREA BASAL, DAP y ALTURA POR SITUACION INTERVENIDA ANTES Y DESPUES DEL RALEO

(superficie por situacion: $3.325 \mathrm{~m}^{2}$ )

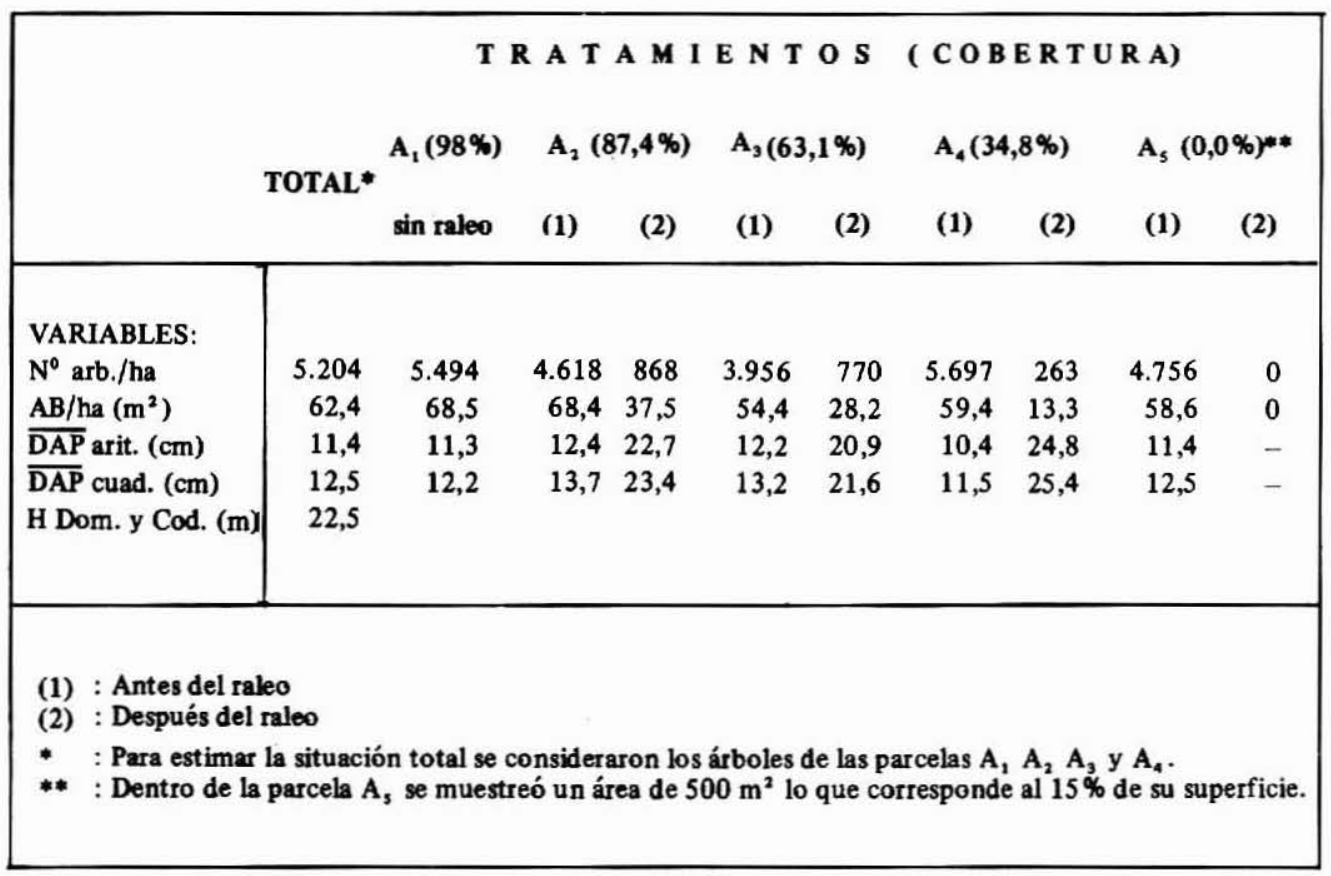

\section{La plantación}

Las plantas de raulí fueron producidas en el vivero del "Centro de Semillas" en Chillán, con semillas recolectadas en Ralco y Colliuco ( $37^{\circ} 53^{\prime}$ Lat. Sur y $72^{\circ} 30^{\prime}$ Long. Oeste) a 700 m.s.n.m. En laboratorio, se obtuvo un $87 \%$ de germinación. 
La siembra se realizó durante la primera quincena de Septiembre de 1985, comenzando la germinación al mes siguiente. Al año de la siembra se realizó el trasplante del material. Entre fines de Octubre y Marzo se cubrió con un sombreadero que dejaba pasar un $50 \%$ de luz. Los fertilizantes aplicados fueron Superfosfato triple (1 aplicación de presiembra con $200 \mathrm{~kg} / \mathrm{ha} \mathrm{y}$ Salitre potásico ( 2 aplicaciones de postsiembra con $400 \mathrm{~kg} / \mathrm{ha}$ ). La extracción del vivero se realizó a fines de Julio de 1987.

Las plantas de roble fueron producidas en el "Centro Experimental Escuadrón" de la empresa "Forestal Mininco S.A.". Las semillas se recolectaron en las áreas de Villarrica, Malalcahuello y Malleco, sin conocerse mayores detalles de su procedencia.

Ensayos de germinación dieron un $69 \%$ para las semillas de Villarrica, un $26 \%$ para las de Malalcahuello y un $47 \%$ para las de Malleco. Se estratificaron en arena húmeda durante 40 días entre $0^{\circ} \mathrm{C}$ y $4^{\circ} \mathrm{C}$.

La siembra se realizó el 01.10 .85 . Al comenzar la germinación las plántulas fueron protegidas con un sombreadero de malla Raschel al 45\%. En Marzo del año 1986 se retiró el sombreadero y se repicaron las plantas. Durante el próximø período estival las plantas se mantuvieron descubiertas.

Las plantas se extrajeron a fines de Junio de 1987 y fueron plantadas durante los primeros días de Julio. Los raulíes se barbecharon durante 6 días y los robles durante 4.

Al fertilizar se aplicó N, P y K. Las aplicaciones se realizaron cuando la planta tenía una altura de $5 \mathrm{~cm}$ y luego en conjunto con la poda de la raíz principal, lateral y el descalce.

Se midieron la altura y el diámetro del cuello, obteniéndose valores medios de $99 \mathrm{~cm} \mathrm{y}$ $9,4 \mathrm{~mm}$ para raulí y $85 \mathrm{~cm}$ y $8,0 \mathrm{~mm}$ para roble.

La fertilización en las parcelas de ensayo se realizó 3 meses después de la plantación, aplicándose Nitrato de Amonio ( $13 \mathrm{~g} /$ planta; contiene $33 \% \mathrm{de} \mathrm{N})$, Superfosfato triple ( $10 \mathrm{~g} / \mathrm{planta}$; contiene $20,1 \%$ de P) y Salitre potásico ( $10 \mathrm{~g} /$ planta contiene $14 \%$ de potasa y $15 \%$ de $\mathrm{N})$. El fertilizante se mezcló y luego se repartió en 3 hoyos dispuestos en triángulo a $5 \mathrm{~cm}$ de profundidad y a $5 \mathrm{~cm}$ de la base de la planta, llenándolos.

\section{RESULTADOS}

A continuación se entregan los resultados obtenidos después del primer período vegetacional en términos de sobrevivencia y crecimiento de las plantas.

\section{La sobrevivencia de las plantas}

Los niveles de sobrevivencia más altos, entre $84 \%$ y $91 \%$, se lograron bajo semisombra (13 $-28 \mathrm{~m}^{2}$ /ha de $\mathrm{AB}$ ). En las condiciones de luminosidad extremas, estos niveles bajaron significativamente, muriendo todas las plantas bajo un $98 \%$ de cobertura (66,5\% de $\mathrm{AB}$ por ha) y el $50 \%$ de éstas expuestas a luz completa sin árboles en el dosel superiot (cuadro 2 ).

La sobrevivencia de las plantas de roble fue significativamente más alta que la de raulí. A luz completa se produjo la manifestación más clara de interacción entre cobertura y especie, sobreviviendo el $64 \%$ de los robles y sólo el $36 \%$ de los raulíes (Tabla 2, figura 2). La fertilización produjo un aumento de la sobrevivencia altamente significativo, correspondiendo a un $17 \%$. Las especies reaccionaron de distinta forma frente a la fertilización. Mientras que para raulí el aumento de plantas vivas fue leve y homogéneo por situación, el roble reaccionó sensiblemente bajo luz completa, aumentando el número de plantas vivas en un $53 \%$ (figura 2 ). 
FIGURA 2

\section{SOBREVIVENCIA RELATIVA DE LA PLANTACION DE RAULI Y ROBLE EN FUNCION DE LA COBERTURA (AREA BASAL) Y LA APLICACION DE FERTILIZANTE DESPUES DEL PRIMER PERIODO VEGETACIONAL}

(Fundo Jauja)

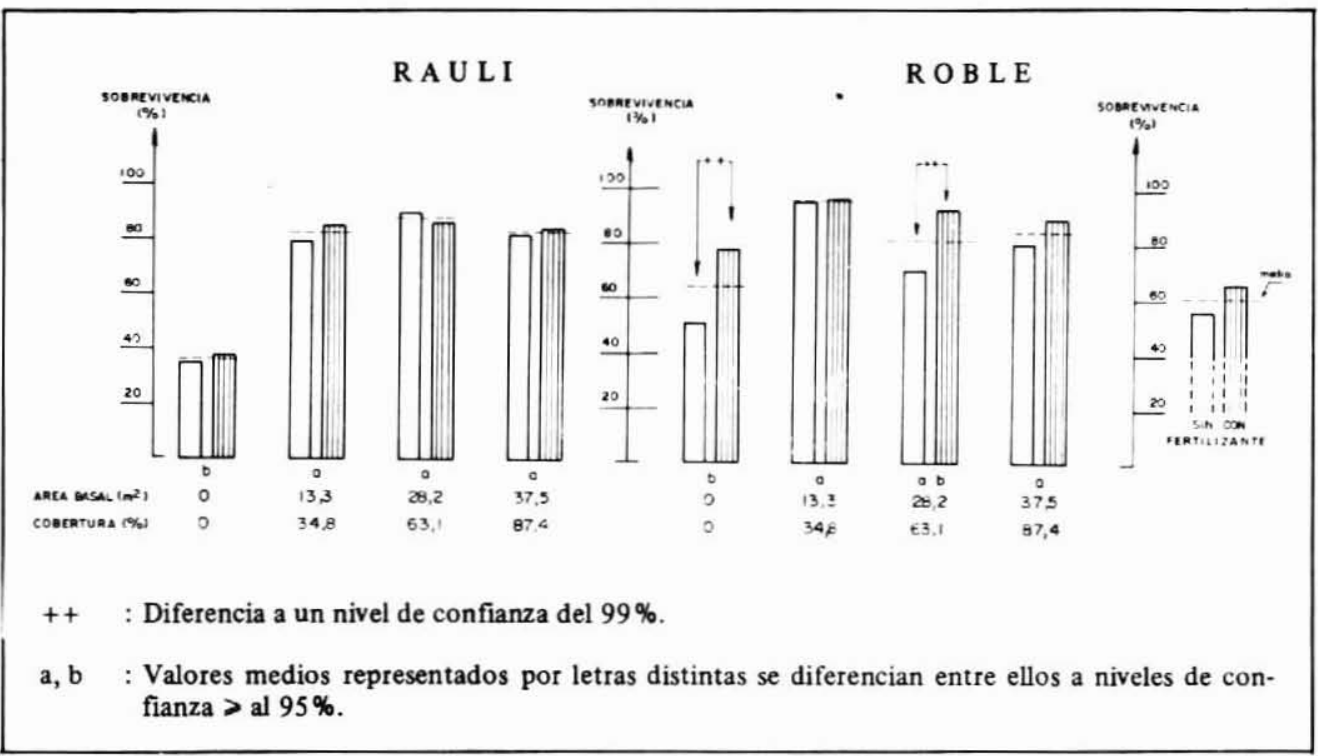

\section{El crecimiento de las plantas}

A continuación se presenta el crecimiento de las plantas empleando las variables de estado, altura, diámetro y un indicador del volumen.

\section{El incremento en altura}

Para el incremento en altura se evaluaron primero todas las plantas y luego se seleccionó el $10 \%$ de éstas, que presentó el crecimiento en altura más alto. Esta depuración del material se realizó con el objetivo de estimar el potencial de crecimiento que se podría lograr con la especie.

A luz completa los incrementos fueron significativamente más altos que en las otras situaciones. Duplicaron a las tres situaciones de semisombra, entre las que, con excepción de un caso para roble, no se pudo detectar diferencia. Los robles crecieron, en promedio un $45 \%$ más que los raulíes.

La fertilización incidió significativamente sobre el crecimiento de ambas especies, superando los individuos con la aplicación en un $56 \%$ a las plantas sin ésta (Tabla 2).

A luz completa y en el rodal más abierto $\left(13 \mathrm{~m}^{2} /\right.$ ha de $\left.\mathrm{AB}\right)$ se lograron las diferencias más espectaculares de crecimiento entre las plantas con y sin fertilizantes (figura 3 ). Para raulí fueron del $68 \%$ y para roble, del $154 \%$ en las parcelas sin cobertura arbórea y del $49 \%$ y $44 \%$, respectivamente bajo la cobertura más suave. 
Al contrario de lo esperado, en la situación con un $63,1 \%$ de cobertura $\left(28,2 \mathrm{~m}^{2} /\right.$ ha de $A B$ ), se produjo un mayor incremento en las parcelas sin fertilización, en relación a aquellas donde se realizó la aplicación. Esto se debió a irregularidades en la cobertura de copas que favorecieron con bastante más luz a las plantas en las parcelas sin la aplicación.

\section{FIGURA 3}

\section{INCREMENTO EN ALTURA DE LAS PLANTAS DE RAULI Y ROBLE EN FUNCION DE LA COBERTURA (AREA BASAL) Y LA APLICACION DE FERTILIZANTE DESPUES DEL PRIMER PERIODO VEGETACIONAL}

(Fundo Jauja)

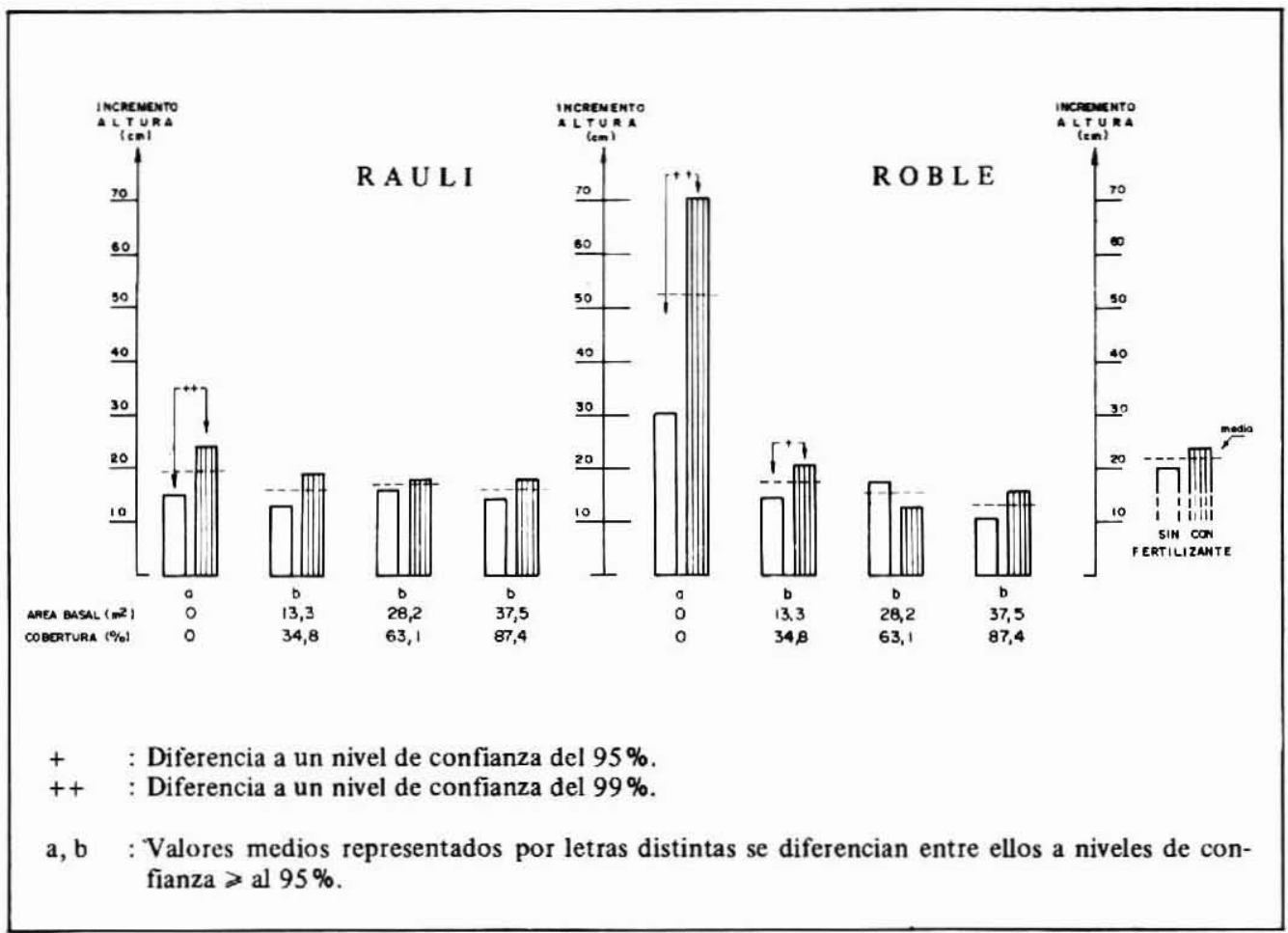

Al seleccionar el $10 \%$ de los individuos de mejor crecimiento, los valores promedio del incremento en altura aumentan sustancialmente. En términos generales los niveles de significancia observados para el promedio del total de los individuos también se mantiene para esta muestra (Tabla 2).

Los incrementos más altos se registraron para las plantas fertilizadas, correspondiendo a los raulíes $41 \mathrm{~cm}$ y $42 \mathrm{~cm}$ bajo la cobertura más suave y sin ésta. Considerando las mismas situaciones de luminosidad, los robles incrementaron $45 \mathrm{~cm}$ y $97 \mathrm{~cm}$ respectivamente (figura 4). 


\section{FIGURA 4}

\section{INCREMENTO EN ALTURA DEL 10\% DE LAS PLANTAS DE RAULI Y ROBLE CON MAS ALTO CRECIMIENTO EN FUNCION DE LA COBERTURA (AREA BASAL) Y LA APLICACION DE FERTILIZANTE DESPUES DEL PRIMER PERIODO VEGETACIONAL}

(Fundo Jauja)

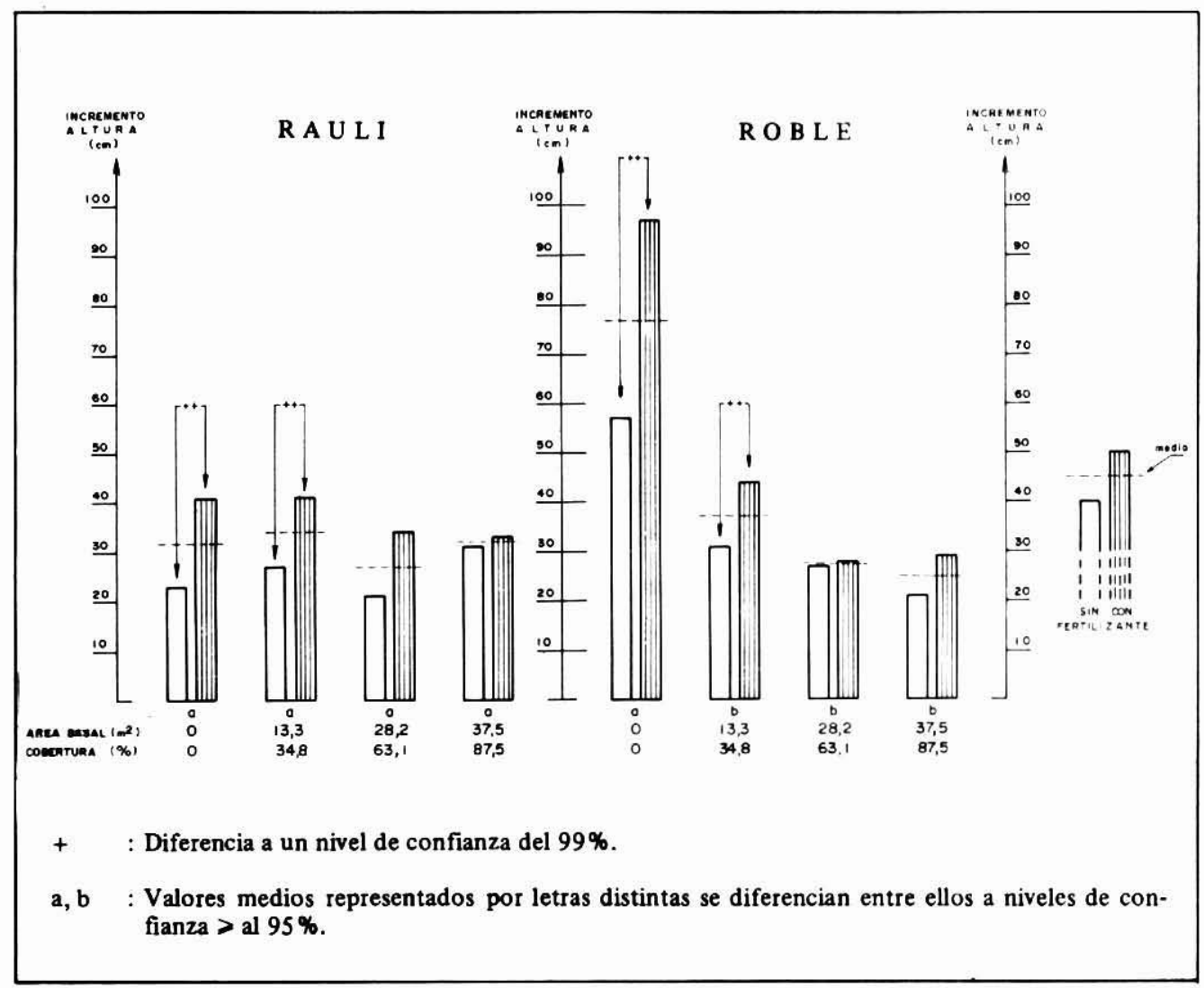

\section{El incremento en diametro}

Los incrementos diametrales más altos, se obtuvieron en las dos situaciones con mayor disponibilidad de luz (AB: $0 \mathrm{~m}^{2}$ y $13 \mathrm{~m}^{2}$ ) (Tabla 2). Esta tendencia fue más clara para el roble, que presenta diferencias entre prácticamente todas las situaciones de cobertura, mientras que para el raulí, éstas no fueron demostrables estadísticamente (figura 5). Bajo todas las variantes de cobertura el crecimiento diametral fue mayor al aplicar fertilizantes. La evidencia más clara de esto se obtuvo en la situación a luz completa, donde los incrementos para raulí y roble superaron al fertilizar en un 59\% y $92 \%$ respectivamente a las plantas sin aplicación (figura 5). 


\section{FIGURA 5}

\section{INCREMENTO EN DIAMETRO DE LAS PLANTAS DE RAULI Y ROBLE EN FUNCION DE LA COBERTURA (AREA BASAL) Y LA APLICACION DE FERTILIZANTE DURANTE EL PRIMER PERIODO VEGETACIONAL}

(Fundo Jauja)

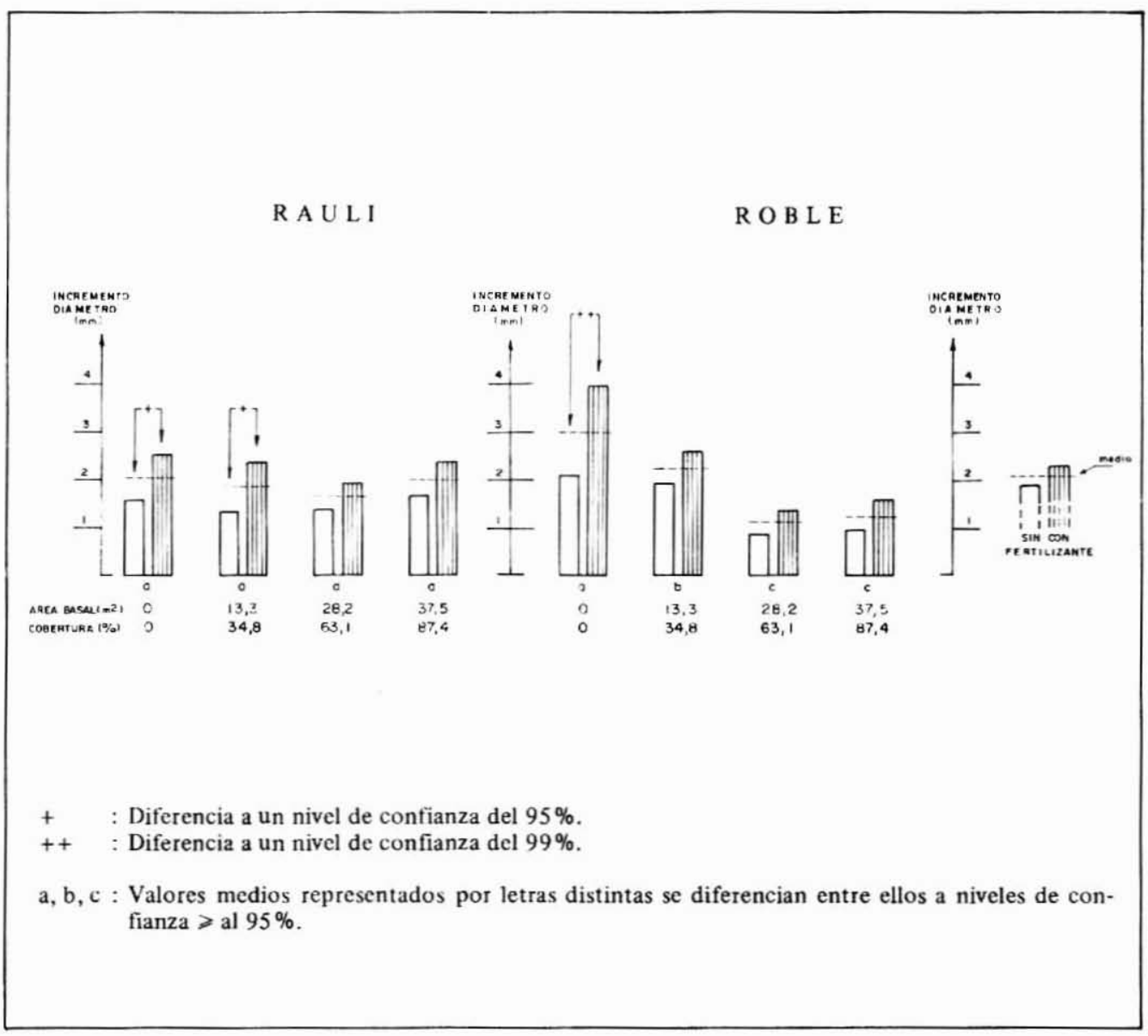

\section{El incremento en volumen}

El incremento volumétrico de las plantas a luz completa casi duplicó al que se obtuvo bajo semisombra. Los raulies crecieron significativamente más que los robles (Tabla 2). Bajo las tres situaciones de semisombra el incremento volumétrico del raulí superó al del roble, mientras que con luz completa esto se invirtió (figura 6). 
FIGURA 6

INCREMENTO EN VOLUMEN DE LAS PLANTAS DE RAULI Y ROBLE EN FUNCION DE LA COBERTURA (AREA BASAL) Y LA APLICACION DE FERTILIZANTE DURANTE EL PRIMER PERIODO VEGETACIONAL

(Fundo Jauja)

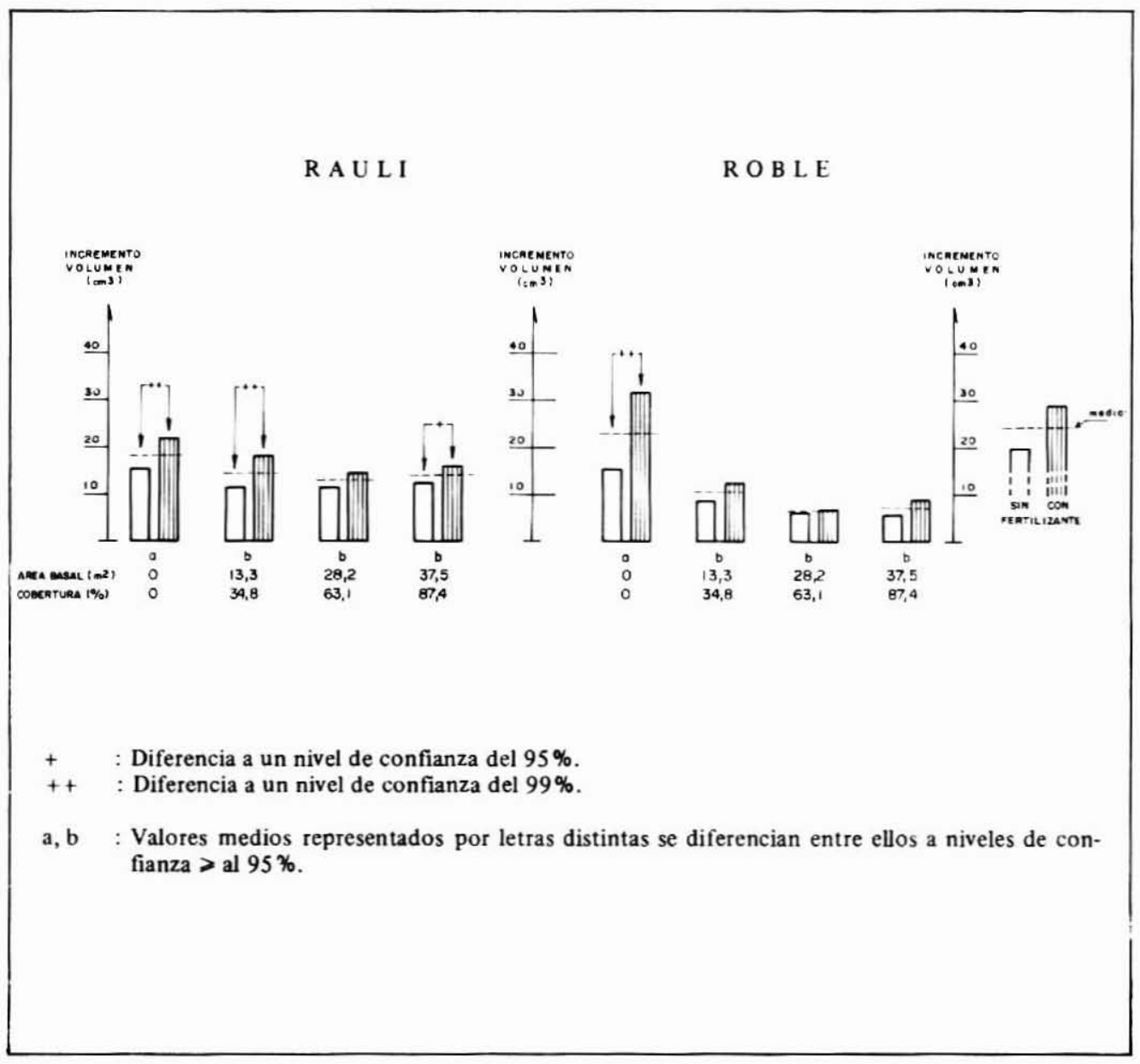

El uso de fertilizante incidió significativamente en un incremento volumétrico más alto. A luz completa la diferencia de crecimiento entre las plantas con y sin aplicación, fue significativa para ambas especies, mientras que bajo semisombra fue más clara para rauli que para roble (figura 6). 
HANS GROSSE W.

TABLA 2

\section{DESARROLLO DE PLANTAS DE RAULI Y ROBLE DURANTE SU PRIMER PERIODO VEGETACIONAL DESPUES DE LA PLANTACION}

(Fdo. Jauja - Agosto 1987 - Abril 1988)

\section{INCREMENTO POR VARIABLE DE ESTADO}

A L T U R A

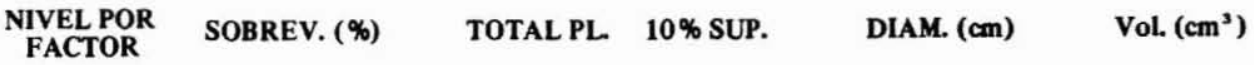

\begin{tabular}{|c|c|c|c|c|c|c|}
\hline \multicolumn{7}{|c|}{ COBERTURA - AREA BASAL (A) } \\
\hline $\begin{array}{r}0,0 \\
34,8 \\
63,1 \\
87,5 \\
98,0\end{array}$ & $\begin{array}{r}0,0 \\
13,3 \\
28,2 \\
37,5 \\
66,5\end{array}$ & $\begin{array}{r}49,8 \mathrm{~b} \\
88,8 \mathrm{a} \\
90,6 \mathrm{a} \\
83,6 \mathrm{a} \\
0,0 \mathrm{c}\end{array}$ & $\begin{array}{c}36,9 \mathrm{a} \\
16,8 \mathrm{~b} \\
15,9 \mathrm{~b} \\
14,6 \mathrm{~b} \\
-\end{array}$ & $\begin{array}{c}54,6 \mathrm{a} \\
35,4 \mathrm{~b} \\
28,5 \mathrm{~b} \\
27,9 \mathrm{~b} \\
-\end{array}$ & $\begin{array}{ll}2,5 & \mathrm{a} \\
2,1 & \mathrm{a} \\
1,4 & \mathrm{~b} \\
1,6 & \mathrm{ab}\end{array}$ & $\begin{array}{c}20,7 \mathrm{a} \\
12,7 \mathrm{~b} \\
9,7 \mathrm{~b} \\
10,3 \mathrm{~b} \\
-\end{array}$ \\
\hline \multicolumn{2}{|c|}{$\begin{array}{l}\text { Nivel de Conf. } \\
\text { (A) }\end{array}$} & ** & $* *$ & ** & $* *$ & ** \\
\hline \multicolumn{7}{|c|}{ ESPECIE (B) } \\
\hline $\begin{array}{l}\text { Rauli } \\
\text { Roble }\end{array}$ & & $\begin{array}{l}60,2 \\
64,9\end{array}$ & $\begin{array}{l}17,0 \\
24,6\end{array}$ & $\begin{array}{l}32,0 \\
42,6\end{array}$ & $\begin{array}{l}1,9 \\
1,9\end{array}$ & $\begin{array}{l}14,8 \\
11,3\end{array}$ \\
\hline \multicolumn{2}{|c|}{$\begin{array}{l}\text { Nivel de Conf. } \\
\text { (B) }\end{array}$} & ** & ** & ** & ** & $* *$ \\
\hline \multicolumn{7}{|c|}{ FERTILIZANTE (C) } \\
\hline $\begin{array}{l}\text { Con } \\
\text { Sin }\end{array}$ & & $\begin{array}{l}57,7 \\
57,4\end{array}$ & $\begin{array}{l}25,3 \\
16,3\end{array}$ & $\begin{array}{l}43,2 \\
31,3\end{array}$ & $\begin{array}{l}2,3 \\
1,5\end{array}$ & $\begin{array}{l}16,2 \\
10,5\end{array}$ \\
\hline \multicolumn{2}{|c|}{$\begin{array}{l}\text { Nivel de Conf. } \\
\text { (C) }\end{array}$} & ** & ** & $* *$ & ** & $* *$ \\
\hline \multicolumn{7}{|c|}{ INTERACCION } \\
\hline$A \times B$ & & * & ** & ** & ** & ** \\
\hline $\mathrm{A} \times \mathrm{C}$ & & ** & ** & $* *$ & ** & *** \\
\hline $\mathrm{B} \times \mathrm{C}$ & & - & $* *$ & - & - & - \\
\hline A $\times B$ & & - & $* *$ & - & - & $* *$ \\
\hline \multicolumn{7}{|c|}{$\begin{array}{ll}\text { * } & \text { : Nivel de confianza }=95 \% \\
\text { ** Nivel de confianza }=99 \% \\
\text { a } & \text { : No significativo } \\
\text { a, b, c } & \text { Valores medios representados por letras minúsculas distintas se diferencian entre ellos a niveles } \\
& \text { de confianza }>\text { al } 95 \% .\end{array}$} \\
\hline
\end{tabular}




\section{DISCUSION}

A continuación se discuten los resultados obtenidos para las plantas de raulí y roble bajo el efecto de los factores cobertura, área basal y fertilizante.

\section{El desarrollo de las plantas en función de la cobertura - área basal}

La producción de biomasa de las plantas depende de su rendimiento fotosintético. La fotosintesis está en función de muchos factores, como son el estado de desarrollo de la planta, el abastecimiento de agua y nutrientes, la apertura de los estomas, la calidad e intensidad de la luz, la temperatura y la concentración del $\mathrm{CO}_{2}$. (ZIEGLER, 1978).

Las distintas intensidades de raleo aplicados al renoval del área en estudio produjeron cambios en los factores que inciden sobre el desarrollo de las plantas.

En el marco del presente estudio no se pudieron evaluar los factores que inciden sobre el desarrollo de las plantas, a excepción de sólo dos variables de estado del rodal como son el área basal y la cobertura. Su utilidad para esto se explica por su alta correlación con la luminosidad y la radiación global (MITSCHERLICH et. al. 1967 y 1981; GROSSE, 1983).

Estos dos indicadores, interpretados en el sentido del largo de onda que posibilita la fotosíntesis o como energía calórica que actúa como catalizador del metabolismo de los árboles, son los más importantes para el crecimiento de las plantas. (BAUMGARTNER, 1960; TURNER y TRANQUILINI, 1961).

Con las distintas situaciones de cobertura probadas, se obtuvieron tal como se esperaba grandes diferencias en la sobrevivencia y el desarrollo de las plantas.

Los niveles de sobrevivencia más altos para los dos Nothofagus ensayados se obtuvieron bajo semisombra entre $13 \mathrm{~m}^{2}$ y $38 \mathrm{~m}^{2}$ de área basal por ha. Estos resultados son coincidentes con los obtenidos en plantaciones de Fagus sylvatica. Se comprobó el mismo fenómeno en un rango de área basal por ha entre $25 \mathrm{~m}^{2}$ y $30 \mathrm{~m}^{2}$ en sectores montañosos de Los Alpes y en el plano con valores de luminosidad relativa entre un 40\% y 50\%. (VON LÜPKE, 1982, GROSSE, 1983).

Para la regeneración natural de Fagus sylvatica SUNER y RÖHRIG (1980) indican una sobrevivencia óptima con $30 \mathrm{~m}^{2}$ de área basal por ha y para Abies alba el LEHRST. f. WALDB. u. FORSTEINR. (1982) un rango entre $30 \mathrm{~m}^{2}$ y $50 \mathrm{~m}^{2}$. En repoblaciones artificiales el comportamiento del Abies alba pareciera ser distinto, ya que al igual que muchas otras especies presenta niveles de sobrevivencia tan altos con luz completa, como bajo semisombra (GROSSE, 1983). Una mayor sobrevivencia bajo semisombra podría ser una característica de los dos Nothofagus probados y del Fagus sylvatica.

El crecimiento en altura, diámetro y volumen de las plantas de raulí y roble aumentan a medida que disponen de más luz. Estos resultados difieren de los obtenidos en vivero donde los mejores incrementos se obtuvieron bajo semisombra (AGUILERA y FEHLANDT, 1981; GROSSE y BOURKE, 1988).

Sobre todo el roble incrementó espectacularmente a luz completa, mientras que bajo semisombra su crecimiento fue bastante más reducido. Los raulíes crecieron a una tasa bastante más pareja bajo todas las condiciones de cobertura.

Es de suponer, que durante los próximos períodos vegetacionales el crecimiento del raulí aumente notoriamente en función de una mayor disponibilidad de luz. Esto, considerando anteçedentes de crecimiento en plantaciones de raulí, Fagus sylvatica y especies del género Abies, que a pesar de ser consideradas como semitolerantes, una vez adaptadas al sitio crecen más al disponer de mayor cantidad de luz. (MAGNUSSEN, 1980; GROSSE, 1983 y 1987). 


\section{El desarrollo de las plantas en función de la aplicación de fertilizante}

El propósito de la fertilización fue el de obtener una orientación general acerca de la reacción de plantas de raulí y roble con y sin aplicación. Los antecedentes de los que se dispone a nivel nacional para poder comparar cantidades y dosificaciones de fertilizante provienen de ensayos realizados con especies forestales exóticas. Por este motivo será indispensable realizar nuevas plantaciones experimentales con rauli y roble en distintos sitios, que consideren diferentes niveles de dosificación y combinación de fertilizantes como también la eliminación de la vegetación competitiva. De este modo, se podrán precisar las cantidades de fertilizante recomendados para cada situación.

La aplicación de fertilizante se realizó en una relación $\mathrm{N}$ : P de $1: 0,35(5,8 \mathrm{~g} \mathrm{~N}$ por 2,0 g P) por planta. La dosis de nitrógeno se acerca bastante a los niveles recomendados por SCHÖNAU y HERBERT (1981) para Eucalyptus en suelos ricos en materia orgánica, superándola en 2,5 g. Sin embargo, la dosis de fósforo aplicada aparece baja según los estándares de SCHÖNAU y HERBERT. Para estas condiciones de suelos, ellos recomiendan la aplicación de nitrógeno y fósforo en una relación 1: 3. En ensayos de fertilización para Eucalyptus en la VII Región, y en zonas áricas, las dosis, superan ampliamente a las utilizadas en Jauja. Las relaciones entre el nitrógeno y fósforo aplicado varían en éstos entre 1: 0,22 y 1:0,35 (PRADO y ROJAS, 1987; PRADO y WRANN, 1988; TORO, 1988). En parte importante de estos ensayos la fertilización tuvo un efecto positivo en el desarrollo de las plantas. Al combinarlas con herbicidas, los crecimientos se mejoraron significativamente.

A pesar de que no se conocen los niveles óptimos de fertilizante que se deben aplicar a plantaciones con raulí y roble, con la dosis utilizada se obtuvieron claras reacciones en su desarrollo.

Sobre todo a luz completa y bajo semisombra suave, las diferencias entre las plantas con y sin fertilizante fueron más notables.

La sobrevivencia fue incrementada significativamente para los robles, mientras que para los raulíes sólo se detectó una tendencia muy leve a reducir la mortalidad.

Todas las variables de estado analizadas de las plantas, incrementaron significativamente con la aplicación de fertilizante. Los niveles de altura se superaron entre un $70 \%$ y un $150 \%$ para raulí y roble respectivamente bajo luz completa. Diferencias en el crecimiento algo inferiores se detectaron bajo semisombra.

El crecimiento obtenido con el fertilizante es de especial importancia para superar la vegetación competitiva. A pesar de no aplicarse herbicidas u otra forma de controi de la competencia, los crecimientos fueron espectacularmente altos. Esto al contrario de otras experiencias que se realizaron en suelos más pobres en materia orgánica y disponibilidad de agua, donde se obtuvieron diferencias de crecimiento significativas sólo al combinar el fertilizante con herbicidas (PRADO y ROJAS, 1987; PRADO y WRANN, 1988).

\section{Proyecciones silvicolas para las plantaciones de rauli y roble}

A pesar de que aún quedan muchas incógnitas por resolver acerca de como lograr el mejor resultado en plantaciones de raulí y robles, es posible entregar algunas recomendaciones generales para el silvicultor. Los resultados presentados no son definitivos para el objetivo propuesto debido a las siguientes limitaciones:

Se desconoce si la procedencia utilizada, como también si las características genotípicas de las plantas, se adecúan al sitio del ensayo y a los objetivos de producción maderera. 
- Aún no existen antecedentes sobre la utilización de herbicidas que actuando sólos o en combinación con fertilizantes puedan mejorar los niveles de sobrevivencia y crecimiento de las plantas de rauli y roble.

- Se desconocen los niveles óptimos de dosificación y combinación de fertilizantes a utilizar, considerando para esto distintas épocas de aplicación.

Los antecedentes de que se dispone actualmente demuestran que se pueden realizar plantaciones exitosas con raulí y roble en áreas con condiciones edafoclimáticas similares a las del fundo Jauja. Para situaciones abiertas, con luz completa aparece con mejores posibilidades de sobrevivencia y crecimiento el roble. Una buena preparación de plantas en vivero, seguida por una adecuada extracción, transporte, plantación y fertilización permitiría lograr un incremento en altura de por lo menos $0,7-1,0 \mathrm{~m}$ durante el primer período vegetativo. Para el raulí la sobrevivencia a luz completa fue bastante más baja. Queda abierta así la investigación acerca de cómo producir plantas en vivero que se adapten de mejor manera a esta condición extrema de luz. A pesar de la baja sobrevivencia del raulí, las plantas fertilizadas llegaron a incrementos de $0,25 \mathrm{~m}$, superando los $0,40 \mathrm{~m}$ al seleccionar el $10 \%$ de individuos de mayor crecimiento. Estos valores se mantienen más o menos en el mismo nivel de incremento bajo la cobertura más suave, igualando el crecimiento del roble. De este modo se podrían obtener crecimientos bastante in. teresantes bajo cobertura suave para ambas especies.

Si bien existe la opción de regenerar los bosques de Nothofagus por la vía natural, para la mayoría de los casos la repoblación artificial cuenta con las siguientes ventajas comparativas.

- En grandes áreas donde los bosques se encuentran empobrecidos respecto a su composición y calidad, la plantación ofrece la posibilidad de modificarlos, aumentando su valor comercial.

- Se facilita la planificación acerca de las intervenciones extractivas al no depender directamente de la periodicidad en la producción de semillas.

- El manejo orientado al establecimiento definitivo, considerando la eliminación de la vegetación competitiva, la fertilización y el crecimiento inicial de la planta, es más sencillo.

- Comenzar la repoblación con plantas grandes, altamente competitivas permite colocarlas en una situación ventajosa con respecto al sotobosque, evitando limpias posteriores.

Esto permitiría manejar el bosque con una sobreposición de estratos, con la ventaja de producir así madera con los árboles maduros y adelantar el comienzo de la rotación siguiente antes de la cosecha final. Este método sería aplicable sólo a situaciones con un número de árboles bajos, que no superen los $15 \mathrm{~m}^{2}$ de área basal. Considerando esta restricción se obtendría un buen crecimiento de las plantaciones y se minimizaría el daño producido a éstas una vez que se realice la cosecha final.

\section{CONCLUSIONES}

Los resultados analizados en el presente documento se deben interpretar dentro del marco de las restricciones que significan el sitio, la procedencia de la planta, el proceso de viverización, la fertilización, la manipulación de una planta determinada y que se trata sólo de antecedentes del primer año después de la plantación.

- La cobertura incide fuertemente en el desarrollo de los individuos:

- Bajo las condiciones extremas se registró la mortalidad más alta correspondiendo al $100 \%$ con una cobertura del $98 \%\left(66,5 \mathrm{~m}^{2}\right.$ de $\left.\mathrm{AB}\right)$ y a una $50 \%$ a luz completa.

- Bajo semisombra ( $35 \%-87 \%$ de cobertura, $\left.13 \mathrm{~m}^{2}-38 \mathrm{~m}^{2} \mathrm{de} A B\right)$ la sobrevivencia fue aproximadamente del $80 \%$. 
- El raulí fue menos tolerante que el roble al ser sometido a luz completa.

- A medida que las plantas disponían de más luz sus crecimientos en términos de altura, diámetro y volumen aumentaron.

La aplicación de fertilizante (N, P, K) aumentó los niveles de sobrevivencia y crecimiento de las plantas.

- En términos significativos la sobrevivencia de los robles aumentó.

- El incremento en altura aumentó en un $70 \%$ para raulí y en un $150 \%$ para roble a luz completa. Aumentó en un $50 \%$ para ambas especies bajo la cobertura más liviana.

- Nuevos ensayos deberán demostrar cuales son los niveles de dosificación y combinación de fertilizantes óptimos.

Se obtuvieron plantas que por su altura se supone van a ser capaces de imponerse frente a la vegetación competitiva.

- Para los raulíes fertilizados la altura alcanzada a luz completa y semisombra leve fue de $1,23 \mathrm{~m}$ y $1,20 \mathrm{~m}$ respectivamente. El $10 \%$ de las plantas con mejor crecimiento llegaron a $1,41 \mathrm{~m}$ y $1,40 \mathrm{~m}$.

- Para robles fertilizados la altura alcanzada a luz completa y semisombra leve fue de $1,60 \mathrm{~m}$ y $1,09 \mathrm{~m}$ respectivamente. El $10 \%$ de las plantas con mejor crecimiento llegaron a $1,81 \mathrm{~m}$ y $1,27 \mathrm{~m}$.

La forestación bajo cobertura leve ( $35 \%$ de cob. $-15 \mathrm{~m}^{2}$ de $\mathrm{AB} / \mathrm{ha}$ ) puede llevar a buenos resultados de crecimiento para rauli y roble, siendo posible disminuir la disponibilidad de luz sólo para el raulí hasta una cobertura del $63 \%\left(28 \mathrm{~m}^{2} \mathrm{de} \mathrm{AB} / \mathrm{ha}\right)$ manteniendo niveles de incremento similares.

Plantar bajo dosel significa manejar el bosque en dos estratos. Los registros de crecimiento y la remoción de los árboles del estrato dominante durante los siguientes años del ensayo, permitirán evaluar integralmente esta posibilidad del manejo en renovales.

En situaciones a luz completa en el sector de Jauja las plantas jóvenes de roble se adaptaron mejor que las de raulí. Esto implica que se deberán buscar opciones de desarrollo mejores para el raulí a través de una preparación especial en vivero y una adecuada selección de su procedencia.

Con el presente estudio queda demostrado que la opción de regeneración del bosque nativo más eficiente y realista es a través de la repoblación artificial. Sólo así se podrá optar por una selección de especies cuantitativa y cualitativa adecuada para enriquecer el bosque y proyectarlo dentro de un marco de rentabilidad económica.

\section{AGRADECIMIENTOS}

El presente estudio ha sido posible gracias al convenio vigente entre Forestal Río Vergara S.A. y el Instituto Forestal.

Se agradece al Señor Alejandro Cuevas (Egresado de Ingeniería Forestal) por su colaboración en la toma de datos de terreno y procesamiento de la información. 


\section{REFERENCIAS BIBLIOGRAFICAS}

1. AGUILERA L. y FEHLANDT, A. (1981): Desarrollo inicial de Nothofagus alpina (Poepp. et Endl.) Oerst., Nothofagus obliqua (Mirb.) Bl., y Nothofagus dombeyi (Mirb.) Bl. bajo tres grados de sombra. Tesis: Universidad Austral de Chile, Fac. de Ingeniería Forestal. 101 pp.

2. BAUMGARTNER, A. (1960): Gelände und Sonnenstrahlen als Standortfaktor am Gr. Falkenstein (Bayerischer Wald); Forstwissenschaftl. Centralblatt, Bd 79. $286-297$.

3. DONOSO C. (1981): Tipos Forestales de los Bosques Nativos de Chile. FO: DP/CH/76/003. CONAF FAO. $70 \mathrm{pp}$.

4. FORVESA (1988): Registros de la estación meteorológica de Jauja.

5. GROSSE, H. (1983); Untersuchungen zur künstlichen Verjüngung des Bergmischwaldes - Ergebnisse eines Forschungsprojektes in den ostbayerischen Kalkalpen. Forschungsberichte d. Forstl. Forschungsanstalt. München 55.203 pp.

6. GROSSE, H. y BOURKE, M. (1987): La regeneración de Raulí. INFOR - CORFO. 84 pp.

7. GROSSE, H. (1987): Desarrollo inicial de plantaciones de raulí. Ciencia e Investigación Forestal. INFOR Santiago. Chile. $\mathrm{N}^{\circ} 1.49-56$.

8. GROSSE, H. y BOURKE, M. (1988): Desarrollo de raulí en vivero bajo distintos niveles de luminosidad y espaciamiento. Ciencia e Investigación Forestal. INFOR - Santiago. Chile. $\mathrm{N}^{\circ} 3.1-12$.

9. LEHRSTUHL FÜR WALDBAU UND FORSTEINRICHTUNG (1982): Bergmischwald Exkursionsführer; Lehrstuhl f. Waldbau u. Forsteinrichtung München. $18 \mathrm{pp}$.

10. LÜPKE, B. von (1982): Versuche zur Einbringung von Lärche und Eiche in Buchenbestände. Habil. Univ. Göttingen. $116 \mathrm{pp}$.

11. MAGNUSSEN, S. (1980): Wachstumsreaktion junger Weiss und Küstentannen verschiedener Herkünfte auf Beschattung. Diss. Univ. Göttingen. 153 pp.

12. MITSCHERLICH, G. KÜNSTLE, E. y LANG, W. (1967): Ein Beitrag zur Frage der Beleuchtungsstärke im Bestande; Allgem. Forstztg., Bd. 138. 213 - 223.

13. MITSCHERLICH, G. (1981): Wald, Wachstum und Umwelt. Frankfurt/Main. Bd. 2. 402 pp.

14. PERALTA M. (1976): Uso, Clasificación y Conservación de suelos. Servicio Agrícola y Ganadero. Stgo. $377 \mathrm{pp}$.

15. PRADO, J.A. y ROJAS, P. (1987): Preparación del sitio y fertilización en el establecimiento de plantaciones de Eucalyptus globulus en la zona semiárida de Chile. Ciencia e Investigación Forestal. Vol. 1; $\mathrm{N}^{\circ} 1.17-28$.

16. PRADO, J.A. y WRANN, J.R. (1988): La importancia de la preparación del sitio y la fertilización en el establecimiento de plantaciones de Eucalyptus. Actas Manejo Silvícola del género Eucalyptus. CORFOINFOR. Junio 1988. Viña del Mar - Chile.

17. SCHÖNAU, A.P.G. y HERBERT, M.A. (1988): Fertilizing Eucalyptus at plantation establishment. Actas Manejo Silvícola del género Eucalyptus. CORFO - INFOR. Junio 1988. Viña del Mar - Chile.

18. SUNER, A. y RÖHRIG, E. (1980): Die Entwicklung der Buchennaturverjüngung in Abhängigkeit von der Auflichtung des Altbestandes. Hannover. Forstarchiv, Bd. 51.145-149.

19. TORO, J. (1988): Efecto de la fertilización en el desarrollo inicial de plantaciones de Eucalyptus. Actas Manejo Silvícola del género Eucalyptus. CORFO - INFOR. Junio 1988. Viña del Mar - Chile.

20. TURNER, H. y TRANQUILINI, W. (1961): Die Strahlungsverhältnisse und ihr Einfluss auf die Photosynthese der Pflanzen. In.: Ökologische Untersuchungen in der subalpinen Stufe; Mitteilungen d. Forstl. Bundes - und Versuchsanstalt Mariabrunn. 69-103.

21. ZIEGLER, H. (1978): Geobotanik. In.: E. Strasburger. Lehrbuch der Botanik. Stuttgart, New York: G. Fischer Verlag, $1.078 \mathrm{pp}$. 